\title{
FACTORES CONTEXTUALES QUE INFLUYEN EN LA PARTICIPACIÓN COMUNITARIA EN ACTIVIDADES DE OCIO Y TIEMPO LIBRE EN PERSONAS EN SITUACIÓN DE DISCAPACIDAD FÍSICA
}

\author{
CONTEXTUAL FACTORS AFFECTING COMMUNITY PARTICIPATION IN LEISURE AND \\ FREE TIME PERSONS WITH DISABILITIES PHYSICAL
}

\section{Mara Arévalo H. ${ }^{1}$ y Jorge Yáñez A. ${ }^{2}$}

\begin{abstract}
Resumen
Se realizó un estudio cualitativo-descriptivo con el objetivo de analizar los factores contextuales que influyen en la participación comunitaria en actividades de ocio y tiempo libre en personas en situación de discapacidad de origen físico con edades comprendidas entre 30 y 59 años y que pertenecen al Departamento de la Discapacidad de Concepción, Chile. La muestra fue seleccionada a través de muestreo por conveniencia y estuvo conformada por cuatro participantes a los cuales se les realizó una entrevista abiertaaplicada de manera individual, durante el período de Junio de 2016.

Como conclusiones, la mayor participación comunitaria en actividades de ocio y tiempo libre de los y las entrevistados/as se encuentra en las actividades provenientes desde programas específicos para personas con discapacidad, en este caso desde el Departamento de la Discapacidad de Concepción, identificándose factores tanto ambientales como personales que influyen en la participación de estas actividades. En relación a los factores ambientales, más de la mitad de los y las entrevistados/as identificó: el espacio físico, el apoyo y relaciones y el clima, como factores influyentes en su participación en este tipo de actividades. En relación a los factores personales, más de la mitad de los y las entrevistados/as identificó: la motivación, el tiempo, los resultados de la experiencia y los factores asociados a la condiciónde salud, como factores influyentes en su participación en este tipo de actividades.
\end{abstract}

\section{Palabras claves:}

factores contextuales, participación comunitaria, ocio, tiempo libre y discapacidad física

\begin{abstract}
A qualitative descriptive study was conducted with the aim of analyzing the contextual factors that influence community participation in leisure activities and free time in persons with disabilities from physical aged between 30 and 59 years and belonging to the Department Disability Concepcion, Chile. The sample was selected by convenience sampling and consisted of four participants who underwent an open interview applied individually during the period of June 2016.

In conclusion, the greater community participation in leisure activities and free time of respondents in the activities arising from specific programs for people with disabilities, in this case from the Department of Disability Concepcion, identifying both environmental and personal factors that influence participation of these activities. In relation to environmental factors, more than half of participants identified: physical space, support and relationships and climate, as factors influencing their participation in such activities. In relation to personal factors, more than half identified: motivation, time, results of the experience and the factors associated with the health condition as factors influencing their participation in such activities.
\end{abstract}

\section{Keywords:}

contextual factors, community participation, leisure, free time and physical disability.

Fecha de recepción: 11/08/2016

Fecha aceptación: 15/11/2016

1 Licenciada en Ciencias de la Ocupación. Telefono: +56982781770 . Correo electrónico: margreen_mara@hotmail.com

2 Licenciado en Ciencias de la Ocupación. Telefono: +56950369761 Correo electrónico: jorgeyanez.araneda10@gmail.com 


\section{INTRODUCCIÓN}

La participación en actividades de ocio y tiempo libre, entendidas según la CIF como: "participar en cualquier tipo de juego, actividad recreativa o de ocio, tales como juegos y deportes informales u organizados, programas de ejercicio físico, relajación, diversión o entretenimiento, ir a galerías de arte, museos, cines o teatros; participar en manualidades o aficiones, leer por entretenimiento, tocar instrumentos musicales; ir de excursión, de turismo y viajar por placer"(OMS, 2001; OPS, 2001).Son actividades queestán inmersas dentro de una comunidad en la cual se desarrollan servicios, programas y actividades de ocio, que pueden desde el sector público, el sector privado con ánimo de lucro o el sector asociativo sin ánimo de lucro (Madariaga y Rubio, s/f).

La importancia de participar en el ámbito del ocio radica en que,a nivel individual éste fomenta la salud y bienestar, es fuente de desarrollo personal y mejora la calidad de vida de las personas (Asociación Iberoamericana de Estudios de Ocio, 1993). En el caso de personas en situación de discapacidad (PsD), el ocio contribuye a su inclusión dentro de la comunidad (Martínez, 2012). Además el ocio presenta beneficios a nivel colectivo, en la medida en que puede crear condiciones que favorezcan el empoderamiento de las comunidades y su desarrollo económico a través del desarrollo de industrias del ocio, bienestar de los ciudadanos y relaciones interpersonales en espacios públicos, desarrollo de un sentido de comunidad y apoyo mutuo entre los miembros de ésta (Guirao y Vega, 2012).Desde un estudio de Terapia Ocupacional realizado por Brown, et al. (2002), en población adulta con discapacidad física, demuestran los beneficios que aporta realizar actividades de ocio, tanto en la esfera mental como física, el placer, el autoconcepto y la participación social.

Sin embargo, en nuestra sociedad actual y producto de nuestra diversidad humana, muchas personas experimentan restricciones en su participación, que finalmente las excluyen de la sociedad. Un grupo de estas personas, son las PsD. Éstas no tienen la misma posibilidad de participar en el ocio como el resto de las personas, debido a la multitud de barreras y a la falta de accesibilidad en muchas actividades que van dirigidas al ocio desde la comunidad (Mesa, 2014).La oferta de ocio actual excluye a las PsD, porque no cumple con las condiciones necesarias para la libre y plena participación. Se dan bajos niveles de participación comunitaria, y persisten las barreras internas y externas que determinan la participación de las PsD en actividades de ocio y tiempo libre (Sevilla, 2012). Esto contribuye a mayores grados de discapacidad.

La discapacidad según la Clasificación Internacional del Funcionamiento, de la discapacidad y de la Salud (CIF), es una construcción simbólica, un término genérico y relacional que incluye condiciones de salud y déficits, limitaciones en la actividad, y restricciones en la participación. Este concepto indica los aspectos negativos de la interacción, entre un individuo con cierta condición de salud y sus factores contextuales, tanto ambientales como personales (OMS, 2001; OPS, 2001).

Por lo tanto, la interacción entre un individuo con cierta condición de salud y sus factores contextuales pueden producir una situación de discapacidad, y por consiguiente limitaciones en actividades y restricciones en la participación.

En este sentido, los factores contextuales son un componente que contribuye sobre lasrestricciones que experimentan las PsD en su participación en el ocio. Por ello, este estudio intenta dar respuesta a la siguiente interrogante: ¿Cuáles son los factores contextuales que influyen en la participación comunitaria en actividades de ocio y tiempo libre de personas en situación de discapacidad física entre 30 y 59 años de edad pertenecientes al Departamento de la Discapacidad de la Ilustre Municipalidad de Concepción?

El objetivo general del presente estudio es: analizar los factores contextuales que influyen en laparticipación comunitaria en actividades de ocio y tiempo libre de los participantes. Sus objetivos específicos son: describir la participación comunitaria en actividades de ocio y tiempo libre de los participantes, identificar los factores ambientales que influyen en la participación comunitaria en actividades de ocio y tiempo libre de los participantes eidentificar los factores personales que influyen en la participación comunitaria en actividades de ocio y tiempo libre de los participantes.

\section{MÉTOdo}

El enfoque de esta investigación será cualitativo, ya que se pretende indagar en las vivencias de las personas tal y como son sentidas y experimentadas por éstas, debido a que son ellas mismas las que más conocen sobre su realidad, utilizando esta información con alcances descriptivos. El diseño a utilizar en esta investi- 
gación será el narrativo, debido a que permite a los participantes narrar sus experiencias, dirigiéndose hacia el entendimiento y al hacer sentido de la experiencia. Además permite contextualizarla en tiempo y lugar, y darle un orden cronológico a la información (Baptista, Fernández y Hernández, 2006).

La población está conformada por personas que presentan una situación de discapacidad física que pertenecen al Departamento de la Discapacidad de Concepción, de sexo masculino o femenino, que tengan entre 30 a 59 años de edad, y que participen de actividades de ocio y tiempo libre en la comunidad. La muestra fue extraída del Departamento de la Discapacidad de Concepción, compuesta por 4 participantes que presentan discapacidad física, 1 hombre y 3 mujeres, entre 30 a 59 años de edad. Ésta fue seleccionada a través de muestreo por conveniencia, y fueron contactados por medio de víatelefónica por parte del psicólogo de la institución.

Los criterios de inclusión son: pertenecer al Departamento de la Discapacidad de la llustre Municipalidad de Concepción, presentar una discapacidad por deficiencia física, tener entre 30 a 59 años de edad, participar de actividades de ocio y tiempo libre en la comunidad. Los criterios de exclusión son: presentar una situación de dependencia severa y presentar una discapacidad por deficiencia distinta a la de índole física.

A cada participante se le entregó un consentimiento informado, explicándoles la finalidad y solicitándoles que de estar de acuerdo firmaran el documento, resguardando cualquier aspecto ético que pudiera ser violado durante la investigación.

Como instrumento para recoger la información, se utilizó una entrevista abiertaaplicada de manera individual, que permitió que cada participante lograra narrar sus experiencias y que el entrevistador/a pudiera manejarla de manera flexible en ritmo, estructura y contenido. Para tener un resguardo de la información la entrevista fue grabada en audio, y para conservar un orden se le pidió a los participantes que describieran a través de una narración cada actividad de ocio mencionando los factores que apoyaban o restringían su participación.

Se realizó un análisis de la información con el fin de alcanzar un mayor conocimiento de la realidad estudiada, siguiendo la estructura de análisis de investigación cualitativa de Martínez (2004). Para ello, los datos fueron transcritos desde audio a texto, para facilitar el análisis de datos.En la reducción de los datos sesepararon unidades, para ello, se diferenciaron unidades temáticas donde cada tema era una determinada actividad de ocio, y unidades por párrafos donde cada párrafo correspondía a una pregunta con su determinada respuesta. Se examinaron las unidades de datos para identificar en ellas determinados componentes temáticos que permitieron clasificarlas en las siguientes categorías de contenido: participación comunitaria, factores ambientales y factores personales. A cada categoría se le asignó un color: rojo, verde y azul respectivamente, para identificar a que categoría corresponde una determinada frase de un párrafo.

Posterior a ello, se realizó una tabla de datos, donde cada fila indica un participante y cada columna una categoría de contenido. De manera que la información de todos los participantes en sus tres categorías quede resumida en una sola tabla. Con la finalidad de facilitar la comprensión de los datos dentro de la tabla, cada frase identificada dentro de las categorías de contenido, fue reemplazada por una palabra que la identificara. En el caso de la categoría factores ambientales, se utilizó la terminología de factores ambientales que propone la CIF.

Una de las principales herramientas utilizadas para obtener conclusiones fue la comparación, que permitió destacar las semejanzas y diferencias entre las unidades de una categoría. La estrategia para la interpretación de resultados y su integración en marcos más amplios que se utilizó en esta investigación fue la aplicación de otras teorías (marcos más generales en los cuales integrar los datos).Para confirmar que los resultados corresponden a los significados e interpretaciones que los participantes atribuyen a la realidad, se utilizó la comprobación con los participantes, que supone contrastar los datos e interpretaciones con los mismos sujetos que constituyen la fuente de esos datos.

\section{Resultados}

\section{Descripción de la Participación Comunitaria en Actividades de Ocio y Tiempo libre}

Los y las entrevistados/as participan en diversas actividades de ocio dentro de la comunidad, las cuales se pueden clasificar como actividades integrativas, entendidas según Arnaiz (2003) y Moriña (2002) citados en Alemañy(2009), como aquellas que insertan de manera parcial y condicional a las PsD en la comunidad; actividades inclusivas entendidas según los mismos autores como aquellas que insertan de manera total e incon- 
dicional a las PsD en la comunidad; y en actividades exclusivas, que son aquellas que no persiguen fines de inserción de las PsD.
La tabla $N^{\circ} 1$, muestra el listado de actividades que realizan los y las entrevistados/as, clasificadas en actividades inclusivas, integrativas y exclusivas.

Tabla 1:

Clasificación de actividades de ocio y tiempo libre.

\begin{tabular}{|c|c|c|}
\hline Actividades inclusivas & Actividades integrativas & Actividades exclusivas \\
\hline Directiva de establecimiento educa- & Yoga & Dirigente de Agrupación de PsD \\
cional & Celebraciones grupales & Ejercicio físico \\
Andar en bicicleta & Taller de costura & Folclore \\
Ir de compras & Terapia de Flores de Bach & Ajedrez \\
Club de fútbol & Taller de manualidades & Taller de manualidades \\
\hline
\end{tabular}

En el listado de actividades inclusivas, la participación es equitativa para todos pudiendo acceder personas que presenten o no algún tipo de discapacidad. Por consiguiente, permite la plena inserción de las PsD en la comunidad.

El listado de actividades integrativas, corresponden a aquellas actividades propuestas por el Departamento de la Discapacidad para PsD, familiares y amigos de la comuna. Sin embargo, sólo participan PsD y familiares de éstos, no evidenciándose la inclusión que tiene como objetivo este departamento. Por este motivo, estas actividades quedan clasificadas sólo como integrativas.

El listado de actividades exclusivas, corresponden a aquellas actividades propias de las agrupaciones de PsD que pertenecen al Departamento de la Discapacidad. Poseen la particularidad que el colectivo de personas que participa de estas actividades son personas que presentan algún tipo de discapacidad.

Se puede apreciar que la mayor participación comunitaria se encuentra ligada a las actividades del Departamento de la Discapacidad, en donde los y las entrevistados/as participan en comunidad, sin embargo, de manera segregada. Es relevante hacer hincapié en esto, debido a que como bien plantea Sierra (2011), existen dos modelos en los que las PsD pueden participar en ocio, la oferta comunitaria y los programas específicos para las PsD. La oferta comunitaria en ocio permite la inclusión de las PsD en la comunidad, mientras que los programas específicos para PsD permiten una participación segregada del resto de la sociedad.

En este estudio son los programas específicos los que toman el protagonismo en la participación de los y las entrevistado/as, siendo la oferta comunitaria utilizada en menor medida. Esto trae repercusiones a nivel individual, debido a que los y las entrevistados/as gozan de una oferta restringida que no promueve la inclusión; y a nivel social, debido a que una comunidad no inclusiva no desarrolla un sentido de apoyo mutuo entre sus miembros.

Antes de que los y las entrevistados/as se integraran a las actividades del Departamento de la Discapacidad, su participación en actividades de ocio y tiempo libre era limitada, y posterior a tener acceso a este departamento, su participación incrementa considerablemente. Un entrevistado comenta que antes de participar de las actividades del centro, prácticamente su única actividad de ocio era ir de compras: "iba a comprar de aburrida porque no, no tenía otra actividad, eso es lo que yo le comentaba" (E. 1, 09 de Junio de 2016). Otro participante, dirigente de una agrupación comenta que a través de la Casa de la Discapacidad tiene acceso a este tipo de actividades: "Claro, yo antes no pertenecía a ningún grupo. Una vez vine aquí porque me llamaron por unas ayudas técnicas, y ahí me dijeron porque no me integraba un grupo aquí, y ahí empecé a venir y ahí ya..." (E. 2, 16 de Junio de 2016). Por otro lado, una de los y las entrevistados/as debido a la exploración de oferta comunitaria en actividades de ocio, descubrió la Casa de la Discapacidad de Concepción: "Ilevaba más de 20 años encerrada entonces ya estaba...", "después ya mi hermana me dijo que me inscribiera en la casa de la discapacidad entonces me inscribí" (E. 3, 09 de Junio de 2016). 
A través de estos discursos, se puede apreciar que la participación de los y las entrevistados/as en actividades de ocio antes de integrarse a los servicios del Departamento de la Discapacidad, aparte de ser limitada, no era satisfactoria. El estar "encerrada" o el "ir a comprar de aburrida", reflejan una deprivación ocupacional a las cuales cualquier persona debiera tener el derecho a acceder. Por su parte, la frase "yo antes no pertenecía a ningún grupo" refleja además una disminuida participación social. En decir, debido a los programas de ocio propuestos por el Departamento de la Discapacidad los y las entrevistados/as han logrado satisfacer sus necesidades de ocio, las cuales no han sido cubiertas de manera efectiva desde la oferta comunitaria en ocio.

Gracias a la creación de los programas específicos para $\mathrm{PsD}$, como son las actividades del Departamento de la Discapacidad, se ha ampliado el abanico de actividades de ocio para este colectivo, entregándoles la posibilidad de escoger bajo sus intereses y necesidades en cuales actividades desean participar. Sin embargo, uno de los roles de este departamento es promover la inclusión de las PsD, esto a través de ofrecer actividades de deporte adaptado, deporte inclusivo y recreación abiertas a la comunidad, donde participen PsD, familiares y amigos de la comuna, lo cual no se ha logrado a cabalidad, debido a la disminuida participación de la comunidad, enfatizando aún más que en estas instancias se produzca una participación segregada, donde sólo se relacionan entre sí personas vinculadas a la discapacidad.

Aunque la cantidad de actividades varía entre los y las entrevistados/as, en todos existe una participación significativa, si se considera que la edad productiva en la que se encuentran limita el tiempo dedicado a estas actividades, debido a que todos desarrollan actividades laborales que sean remuneradas o no, dejan un limitado tiempo para participar de otras ocupaciones. Un entrevistado comenta que aunque tiene poco tiempo debido a su rol de trabajador, arregla sus turnos para poder asistir a las actividades de su agrupación todas las semanas: "Los otros días yo voy a trabajar porque no tengo tiempo, a veces me cambian los turnos, me toca en la mañana, me toca en la tarde, por eso yo los días jueves me las arreglo para poder estar aquí con mi grupo" (E. 2, 16 de Junio de 2016).

De acuerdo a la clasificación de actividades de ocio y tiempo libre que propone la CIF (Organización Mundial de la Salud, 2001), se identifica participación de los y las entrevistados/as en actividades de juego, deporte, cultura, manualidades y socialización. Cabe destacar que todos participan de actividades manuales y de socialización, y que las actividades de juego y deportivas están más ligadas al sexo masculino, en este estudio.

En relación a las actividades de socialización, los y las entrevistados/as se relacionan principalmente con personas que están ligadas con la discapacidad, lo cual además de producir la segregación de la cual se ha hablado, limita la posibilidad de sensibilizar al resto de la sociedad sobre esta temática. A continuación se citan a entrevistados que se refieren al tema: "Todos tienen algún tipo de discapacidad pero de diferencias en discapacidad" (E. 2, 16 de Junio de 2016); "Somos como dos o tres que tenemos una discapacidad física, el resto de las otras personas no tienen discapacidades físicas, pero son familiares de personas con capacidades diferentes" (E. 1, 09 de Junio de 2016).

Para terminar, como menciona Sevilla (2012), la oferta comunitaria de ocio sigue excluyendo a las PsD porque no cumple las condiciones necesarias para la libre y plena participación, siendo tratadas como un objeto de intervención y colectivo receptor de programas, lo cual se puede visualizar en el presente estudio. Esto es contradictorio a la premisa que la comunidad debe satisfacer las necesidades de todas las personas independientemente de sus particularidades y condiciones, planteada desde un Enfoque de Derechos Humanos (Fondo de las Naciones Unidas para la Infancia, 2005). Por su parte, la política nacional menciona que "es la sociedad la que debe hacer las adecuaciones para incluir a las personas con discapacidad, eliminando esas barreras y evitando esas restricciones que impiden a las personas con discapacidad estar en igualdad de condiciones con las demás" (Servicio Nacional de la Discapacidad, Ministerio de Desarrollo Social, 2013, p.4), siendo uno de sus objetivos "promover el bienestar físico, espiritual y social de las personas con discapacidad a través de su participación en actividades culturales, deportivas y recreativas tanto públicas como privadas" (SENADIS y Ministerio de Desarrollo Social, 2013, p.20). De lo anterior, podemos decir que es la comunidad la responsable de la exclusión de las PsD y de que deban encontrar otros medios de participación en ocio.

Sin embargo, a través de los programas específicos para PsD, los y las entrevistados/as han logrado involucrarse en una variedad de actividades comunitarias de ocio y tiempo libre, que les ha permitido satisfacer sus necesidades de ocio. Esto tiene un impacto positivo sobre la salud de éstas personas, en la medida en que desde la terapia ocupacional se reconoce que el compromiso con actividades que permiten la participación 
deseada en la comunidad contribuye a alcanzar y mantener la salud de las personas (Ávila, et al., 2008).

\section{Factores ambientales}

Se identificaron influencias desde los tres tipos de ambientes que propone la CIF: físico, social y actitudinal. Cabe destacar, que todos estos factores ejercen efectos facilitadores; sin embargo, algunos de ellos ejercen también un efecto de barrera en la participación comunitaria en actividades de ocio y tiempo libre de los y las entrevistados/as.

La tabla $N^{\circ} 2$, muestra los factores ambientales que influyen en la participación comunitaria en actividades de ocio y tiempo libre de los y las entrevistados/as, definidos como barreras y/o facilitadores.

Tabla 2:

Factores Ambientales por tipos de efecto.

\begin{tabular}{|l|l|}
\hline Barreras & $\begin{array}{c}\text { Espacio físico, actitudes sociales, sistema de organización, clima y } \\
\text { transporte. }\end{array}$ \\
\hline Facilitadores & $\begin{array}{c}\text { Espacio físico, actitudes sociales, apoyo y relaciones, equipamien- } \\
\text { to, servicios de oferta comunitaria, sistema de organización, cli- } \\
\text { ma, transporte, servicios de oferta comunitaria y ayudas técnicas. }\end{array}$ \\
\hline
\end{tabular}

A continuación se describirá cada factor y su influencia sobre la participación comunitaria en actividades de ocio y tiempo libre.

El factor apoyo y relaciones, entendido como la cantidad de apoyo, tanto físico como emocional, proporcionan otras personas o animales (OMS, 2001; OPS, 2001), se identificó como un factor facilitador en todos los y las entrevistados/as, a causa del constante apoyo que recibían por parte de sus compañeros y monitores en sus actividades de ocio y tiempo libre. Así lo refiere uno de los y las entrevistados/as al realizar la actividad de ajedrez: "el mismo profesor los apoya, que sigamos, vayan aprendiendo" (E. 2, 16 de Junio de 2016). Otro entrevistado refiere recibir ayuda en la actividad de Yoga: "la monitora eh igual les va dando ideas, para que a uno no se le complique la vida haciendo un movimiento" (E. 1, 09 de Junio de 2016).

El espacio físico es un factor facilitador de la participación en la medida en que su disponibilidad y sus características tales como tamaño y temperatura apoyan la participación de los y las entrevistados/as en sus actividades de ocio. Sin embargo, se trasforma en un factor barrera cuando no hay espacios disponibles o cuando sus características interfieren con la actividad. Ejemplos de esto son espacios donde se realizan actividades con temperaturas frías que no cuentan con calefacción: "Sí, sí porque no hay...tienen una estufa que está pegada a la pared, pero no funciona" (E. 1, 09 de Junio de 2016).
Y salas de tamaño pequeño para grupos grandes: "esta es la sala más grande que tiene la casa de la discapacidad, el resto de las salas son muy pequeñas y no alcanza, sobre todo para costura que se ocupan máquinas, se ocupan mesas amplias, que es único lugar donde se puede trabajar" (E. 1, 09 de Junio de 2016). Cabe destacar, que la influencia de este factor es por motivos que pueden interferir en la participación de cualquier persona y no sólo de PsD.

El clima, entendido como características y aspectos meteorológicos, es identificado como un factor barrera pero también como facilitador. Dentro de sus diversas características como temperatura, presión atmosférica y precipitaciones, influye de diferentes maneras. Por ejemplo, en un entrevistado las temperaturas bajas y precipitaciones favorecían la actividad: "si llueve se supone que uno debería tener más dificultad por la lluvia, yo no tengo dificultad (ríe), me cuesta menos, si el día es demasiado cálido yo voy a tener mucho más dificultad en llegar" (E. 1, 09 de Junio de 2016). En otro entrevistado esta misma característica interfería en la actividad: "cuando llegue el tiempo bueno ya ahí vamos a... porque en este tiempo, para sacar a los chiquiIlos para allá, algunos son bien delicaditos, se resfrían" (E. 2, 16 de Junio de 2016). Por lo tanto, el clima ejerce influencias que varían en función de una persona con una determinada condición de salud a otra y del contexto en el que se realice la actividad. 
Las actitudes sociales, entendidas como opiniones y creencias mantenidas por personas de una determinada cultura, sociedad, subcultura u otro grupo social, sobre otras personas o sobre otras cuestiones sociales, políticas y económicas, que influyen en el comportamiento y las acciones grupales o individuales (OMS, 2001; OPS, 2001), fueron identificadas por dos de los y las entrevistados/as como un factor influyente en su participación. En un entrevistado las actitudes de su familia ejercían barreras sobre su participación en actividades de ocio, debido a que no confiaban en sus habilidades para realizar estas actividades, "si a mí me prohíben para salir a participar pero voy igual nomas cuando teníamos que participar, nunca falte...", "porque ellos son así conmigo, me discriminan siempre en la casa..." (E. 3, 09 de Junio del 2016). Además, este mismo participante percibía como barrera las actitudes de algunos compañeros con los que realiza actividades de ocio, "a veces llega una niña que es un poco inquieta pero no la..." (E. 3, 09 de Junio del 2016)"no la toma en cuenta nomas..." (Entrevistador, 09 de Junio del 2016), "no..." (E.3, 09 de Junio del 2016). Las actitudes de los extraños también han sido identificadas como influyentes, pero a diferencia de las anteriores, ejercen un mayor efecto facilitador para la participación en el ocio, un entrevistado refiere que la mayoría de las personas extrañas eran amables cuando PsD necesitaban algún tipo de ayuda, sin embargo, existían personas que eran indiferentes y en ocasiones hostiles ante situaciones como esta: "Si yo les pido me guardan puesto, siempre me han ayudado, sea la persona que sea siempre me ha ayudado a que pueda... ellos ven que yo me voy a sentar y me guardan el puesto, siempre ha sido así, hay otras personas que me dicen: pero pase para adelante, me dicen; pero hay otras personas que son inconscientes, que: no, yo primero" (E. 1, 09 de Junio de 2016). Como podemos observar sigue existiendo discriminación hacia las PsD, lo cual interfiere con la plena participación y desempeño en la comunidad.

Los servicios representan la provisión de beneficios, programas estructurados y operaciones, en varios sectores de la sociedad, diseñados para satisfacer las necesidades de los individuos, los cuales pueden ser adaptados y especialmente diseñados (OMS y OPS, 2001); fueron identificados entre los y las entrevistados/as como un factor facilitador de la participación. Sin embargo, en el relato en donde se alude a este factor, principalmente se refieren a la participación en actividades del Departamento de la Discapacidad. Las actividades que se ofrecen desde éste departamento se adaptan a las diversas necesidades de las personas que participan, facilitando el equipamiento necesario para su ejecución, generando un servicio de calidad y que por lo tanto, más personas se adhieran a participar. Pero, ¿qué pasa con la oferta comunitaria en ocio para la población general? Como se menciona en el análisis de la participación comunitaria, la participación en actividades que ofrece la oferta comunitaria en ocio es significativamente menor. Entonces, se puede cuestionar si los servicios que propone la oferta comunitaria, cumple con las condiciones necesarias para facilitar en alguna medida la participación.

Los sistemas de organización, entendidos como sistemas que están diseñados para organizar, controlar y supervisar los servicios que proporcionan beneficios y programas (OMS y OPS, 2001), como por ejemplo los sistemas utilizados en la organización de horarios y distribución de salas para talleres con sus respectivos monitores, son un factor que en ocasiones actúan como barrera para la participación, debido a que una organización deficiente interfiere en el funcionamiento de las actividades. Los y las entrevistados/as refieren que en reiteradas ocasiones sus actividades son suspendidas 0 deben realizar un cambio dentro de la rutina normal ya que las salas que generalmente ocupan son facilitadas para otras reuniones, los monitores deben suspender actividades porque deben asistir a otros compromisos o agendan actividades extraprogramáticas para el mismo día en que realizan sus actividades normales. Así lo relata uno de los y las entrevistados/as: "Sí, pero igual se está conversando eso, de que pudiera ser un día diferente, en el cual todo el grupo pudiera asistir y la profesora igual pudiera asistir", "Para no ocupar nuestro día, porque ese día para nosotras, o sea para la mayoría de nosotras que hacemos yoga es importante, porque es un solo día a la semana" (E. 1, 09 de Junio de 2016).

El equipamiento es identificado como un factor facilitador por los y las entrevistados/as, debido a que algunas actividades necesitan de equipos que son facilitados por la institución que ofrece los servicios, en este caso el Departamento de la Discapacidad en colaboración con la Municipalidad de Concepción. En este factor se incluyen los equipos que son de ayuda para PsD, que también fueron identificados como facilitadores de la participación: "a veces aquí vienen dos kinesiólogos a hacernos ejercicios, nos sacan al parque, porque tienen unas bicicletas de dos, que andan dos, y uno parte para el parque, lo hacen andar, hacer ejercicio, no sé, hacen cualquier actividad con nosotros" (E. 2, 16 de Junio de 2016), en este ejemplo, 
se puede evidenciar la utilidad que puede prestar un equipo que, si bien, puede ser utilizado por cualquier persona, ya que no es especialmente diseñado para PsD, puede apoyar la participación que se ve restringida por deficiencias físicas que no le permiten a estas personas utilizar una bicicleta convencional. En cuanto al equipamiento de las actividades de oferta comunitaria, este sigue siendo escaso.

El trasporte se identificó como un factor barrera, cuando se trata de buses y microbuses del trasporte público, debido a que no cuenta con las medidas de accesibilidad necesarias para que las PsD puedan hacer uso de él de manera efectiva; y como factor facilitador, cuando las características del vehículo privado, taxi o colectivo son más accesibles permitiendo su uso sin mayores dificultades. A modo de ejemplo se cita a un entrevistado: "Por donde yo vivo, pasan buses y colectivos, yo tengo más problemas con los buses, para subirme no, pero para bajarme si po, me cuesta arto, y me da miedo caerme, asique por eso ocupo eh ocupo un colectivo" (E. 1, 09 de Junio de 2016). Si bien, el trasporte no es un factor propio de las actividades de ocio, las personas necesitan utilizarlo para movilizarse desde sus hogares a los diferentes lugares donde realizan sus actividades de ocio y tiempo libre. El no contar con un medio de trasporte accesible produce finalmente que las $\mathrm{PsD}$ experimenten restricciones en su participación en comunidad.

Las ayudas técnicas, entendidas como cualquier producto, instrumento, equipo o sistema técnico utilizado por una persona con discapacidad, creado específicamente para ello o de uso general, que sirva para prevenir, compensar, supervisar, aliviar o neutralizar la discapacidad (OMS y OPS, 2001); se identificaron como factor facilitador en los y las entrevistados/as. Dos de los y las entrevistados/as identificaron este factor como un apoyo para su participación en actividades de ocio: "Si yo camino una cuadra sin el bastón y me empieza a doler todo, me empieza a doler la cadera, me empiezan los dolores en los músculos de las piernas y me da fatiga" (E. 1, 09 de Junio de 2016), "Yo tengo mi tendón de Aquiles más corto, pero yo tengo que usar un zapato con realce eso no me complica, yo ando igual en bicicleta, no me molesta" (E. 2, 16 de Junio de 2016). Si estas personas no contaran con su bastón y su zapato con realce, respectivamente, se les dificultaría realizar sus actividades, el contar con ayudas externas que compensen sus limitaciones apoya la participación en actividades de ocio.

\section{Factores personales}

Se identificaron lo siguientes factores que influyen en la participación comunitaria en actividades de ocio y tiempo libre de los y las entrevistados/as: motivación, tiempo, resultados de la experiencia, factores asociados a la condición de salud, rango de la emoción (ansiedad), recursos económicos personales y Funciones de la atención, específicamente concentración.

Desde la CIF los efectos que producen los atributos de una persona no aplican como facilitadores ni como barrera. Sin embargo, se reconoce que estos factores ejercen influencias, las cuales en alguna medida van a contribuir a favorecer o restringir la participación en actividades de ocio y tiempo libre.

A continuación se describirá cada factor y su influencia sobre la participación comunitaria en actividades de ocio y tiempo libre:

La motivación por realizar este tipo de actividades es identificada por los y las entrevistados/as como uno de los factores más importantes que influye en su participación, es el punto de partida para participar en una actividad. Según el discurso de un entrevistado, es esencial y primordial para involucrarse en este tipo de actividades: "Es sólo tener las ganas de hacer, porque no todos tienen que hacerlo igual, de la misma manera el movimiento o el ejercicio, es como tú puedas, porque ahí por ejemplo yo tengo una discapacidad física y me cuesta hacerlo igual que las demás, pero igual lo hago" (E. 1, 09 de Junio del 2016). Lo cual coincide con la teoría, ya que según la Asociación Americana de Terapia Ocupacional (AOTA), ocio y tiempo libre es "una actividad no obligatoria que esta intrínsecamente motivada" (AOTA, 2008).

El tiempo que tienen los y las entrevistados/as para realizar este tipo de actividad es otro factor identificado como influyente en la participación, el cual es limitado y en ello también hay que considerar la edad productiva en la que se encuentran, según resultados de la ENDISC II la tasa de participación laboral de las PsD entre las edades de 30-44 años es de un $72,9 \%$ y de un $65,1 \%$ entre las edades de 45-59 años (Ministerio del desarrollo Social, 2015). Si bien algunos de los y las entrevistados/as cumplen con una jornada laboral, los demás entrevistados cumplen con una cantidad de roles, los que a su vez son altamente demandantes. Todos los y las entrevistados/as realizan distintos roles dentro de su rutina, por lo que la distribución de su tiempo es totalmente heterogénea unos de otros. Tres de los y 
las entrevistados/as identificaron el factor tiempo como una limitante en la participación en actividades de ocio y tiempo libre, ya que dos de ellos cumplen una jornada laboral de 48 horas semanales y también otros roles: "yo trabajo de lunes a viernes acá, entro a las ocho y media y salgo a las seis de la tarde, me queda sábado y domingo nomas" (E.4, 09 de Junio del 2016); "Los otros días yo voy a trabajar porque no tengo tiempo, a veces me cambian los turnos, me toca en la mañana, me toca en la tarde, por eso yo los días jueves me las arreglo para poder estar aquí con mi grupo" (E. 2, 16 de Junio de 2016). Otro entrevistado que se encarga de las labores del hogar y del cuidado de sus hijos, menciona: "Yo, a veces (ríe), cuando tengo tiempo, no, no puedo... o sea si yo pudiera iría a todas las actividades, pero como soy madre, dueña de casa, no siempre se puede"(E.1, 09 de Junio del 2016).

Los resultados de la experiencia de participar en este tipo de actividades promueven que la participación en estas actividades se mantenga, debido a que las personas se relajan, aprenden cosas nuevas, comparten con otros, algunas actividades ejercen un efecto terapéutico que contribuye a la mantención de la salud. Como ejemplo, se cita a un entrevistado: "El yoga me ha ayudado a no sentirme tan atrofiada porque mi enfermedad aparte de que es, cuesta hacer movimientos normales, es dolorosa y por eso escogí yoga, porque me ayuda a que mis músculos ya no duelan tanto como antes, ayuda arto, arto, arto. Y más encima eh me ha ayudado a relajarme más" (E. 4, 09 de Junio del 2016).

Los factores asociados a la condición de salud son un factor que restringe la participación en el ocio. Entre ellos, se encuentran la fatiga y el dolor. En algunos casos estos factores son una limitante a la hora de poder completar las actividades de ocio y tiempo libre, además de interferir el desempeño de estas actividades. Como ejemplo citamos a un entrevistado: "Ya a las dos horas ya estoy reventada, ya estoy muy cansada y me quiero ir a la casa (ríe), ya el cuerpo no me da más de dos horas"; "y no es que termine cansada, sino que empiezo con dolores, esa es la cuestión, empiezo con dolores de cadera y hasta ahí no mas llego po"(E. 1, 09 de Junio del 2016). Aun así, estos factores no son una condicionante para no realizar actividades de ocio y tiempo libre. Sin embargo, limitan ciertas actividades que demandan una mayor capacidad física.

Otros de los factores personales que influyen en la participación de actividades de ocio y tiempo libre en la comunidad, que fueron identificados sólo en el entrevistado $\mathrm{N}^{\circ} 1$ son: la ansiedad, lo cual según relata la entrevistada, requiere de un tratamiento: "Pero es por eso que yo al psicólogo le pedía algo para la ansiedad, porque es una ansiedad, el ir a andar comprando de aburrida" (E. 1, 09 de Junio del 2016); los Recursos económicos personales, debido a la ansiedad de comprar cosas, señalando: "si la plata, me falta plata" (E. 1, 09 de Junio del 2016); y la concentración, considerándola como dentro de las competencias básicas para realizar de manera efectiva la actividad: "ahí lo importante es la concentración, el que tú puedas respirar bien y hacer las respiraciones bien hechas, eh te va a ayudar más." (E. 1, 09 de Junio del 2016).

\section{DISCUSIÓN}

Desde el presenta estudio, se concluye que los y las entrevistados/as participan de manera significativa en diferentes actividades de ocio y tiempo libre en la comunidad. Sin embargo, la mayor participación se encuentra en las actividades integrativas y exclusivas para PsD, provenientes desde el Departamento de la Discapacidad Concepción. En menor medida participan de actividades inclusivas provenientes de la oferta comunitaria en ocio, los motivos de esta disminuida participación no se profundizan debido a que escapan de los objetivos del estudio.

Si bien, el Departamento de la Discapacidad ofrece una variedad de actividades que satisfacen las necesidades de ocio de los y las entrevistados/as, no se logra evidenciar inclusión en la participación de estas actividades, debido a que sólo existe participación de personas vinculadas a la discapacidad y el resto de la comunidad no se hace partícipe de las instancias de participación en actividades de ocio abiertas a la comunidad que ofrece este departamento, con la finalidad de favorecer procesos inclusivos. Esta situación favorece la participación segregada de los y las entrevistados/as.

De acuerdo a la identificación de los factores ambientales que influyen en la participación comunitaria en actividades de ocio y tiempo libre de los y las entrevistados/as, se encontraron tres factores ambientales identificados como influyentes por más de la mitad de los y las entrevistados/as: el apoyo y relaciones, el espacio físico y el clima. Para este estudio el apoyo y relaciones actúa como el principal factor ambiental que facilita la participación de los y las entrevistados/as en 
este tipo de actividades. El espacio físico y el clima actúan principalmente como barreras para la participación de los y las entrevistados/as en estas actividades.

Si bien, existe una mayor cantidad de factores ambientales que ejercen un efecto facilitador, la influencia de los factores que están poniendo barreras a la participación es significativa y determinante para los y las entrevistados/as, a tal punto que siguen experimentando exclusión en su participación en el ocio. Sigue predominando la participación en instancias segregadas al resto de la comunidad.

De acuerdo a la identificación de los factores personales que influyen en la participación comunitaria en actividades de ocio y tiempo libre de los y las entrevistados/as, se encontraron cuatro factores identificados como influyentes por más de la mitad de los y las entrevistados/as: la motivación como un factor primordial para la participación, el tiempo como un requisito que deben tener las personas para poder participar, los resultados de la experiencia en donde si se tienen resultados positivos las personas seguirán involucrándose en estas actividades, y los factores asociados a la condición de salud en donde problemas en las funciones o estructuras corporales producían una limitación en estas actividades. Los primeros tres factores tienen la característica de ser transversales a todas las personas, es decir, no influyen exclusivamente a las PsD.

La solución propuesta para las limitaciones $q u$ e actualmente presentan los y las entrevistados/as en relación a su participación en actividades comunitarias de ocio y tiempo libre, sería la promoción de actividades de ocio inclusivas desde los programas para PsD en conjunto con los servicios de oferta comunitaria regular. A través de este trabajo en conjunto promover la inclusión de las PsD, considerando los factores que están interfiriendo en esta plena participación. Además de las necesidades, intereses y características de las propias PsD.

Para lograr una participación en actividades de ocio mas inclusivas, es necesario que los organismos que trabajan en materia de discapacidad, refuercen los conceptos de igualdad de oportunidades e inclusión social en el ámbito del ocio, y que no sólo se enfaticen estos conceptos en el ámbito de la educación y el trabajo, ya que el ocio es una importante área que promueve la salud y el desarrollo personal de PsD. Además, es necesario incluir a la población adulta en la variedad de programas de ocio para las PsD y no sólo centrar la atención en el colectivo de especial protección o en riesgo social, correspondiente a menores, jóvenes y adultos mayores, debido a que este grupo etario par- ticipa de roles productivos que deben ser equilibrados con actividades no obligatorias tendientes al disfrute y satisfacción personal.

En este sentido, la terapia ocupacional como disciplina tiene el compromiso de promover la salud a través de la ocupación, pero no sólo se centra en la ocupación como tal, sino también en los factores que influyen en ella. Como ejemplo, citamos un programa realizado en Canadá (Van, 2009), para promover la participación y la satisfacción en las actividades de ocio de personas con discapacidad física, que demostró que en los participantes que recibieron la intervención de Terapia Ocupacional se observó un aumento en la satisfacción con el ocio, el tiempo destinado a las actividades de ocio activo y en el número total de actividades lúdicas desempeñadas. Esto da cuenta del rol esencial que desempeña la Terapia Ocupacional en el ámbito del ocio.

Por último, se sugiere que para próximas investigaciones se profundice en la disminuida participación de las PsD en los servicios de la oferta comunitaria regular en ocio, con la finalidad de poder analizar estos aspectos y aportar a la reformulación de los programas y proyectos existentes.

\section{REFERENCIAS BIBLIOGRÁFICAS}

Alemañy, C. (2009). Integración e Inclusión: Dos Caminos Diferenciados en el Entorno Educativo. Cuadernos de Educación y Desarrollo, 1(2), 23. Recuperado de http://www.eumed.net/rev/ced/02/cam5. htm

American Occupational Therapy Asociation. Occupational therapy practice framework: and process ( $2^{\text {nd }}$ ed.). Am J Occup Ther. 2008; 62: 625-683.

Asociación Iberoamericana de Estudios de Ocio. (1993). Asociación OTIUM. Recuperado de: http://www.asociacionotium.org/wpcontent/uploads/2009/03/carta-de-la-educacion-del-ocio.pdf

Baptista, P., Fernández, C., y Hernández, R. (2006). Metodología de la Investigación. México: McGraw Hill. Recuperado de: https://competenciashg.files.wordpress.com/2012/10/sampieri-et-al-metodologia-de-la-investigacion-4ta-edicion-sampieri-2006_ocr.pdf

Brown, E.,Foris, C., King, G., Specht, J.(2002). The importance of leisure in thelives of persons with congenital physical disabilities.American Journal of OccupationalTherapy,56(4), 436 - 445. Disponible en: http://ajot.aota.org/data/Journals/AJOT/930142/436.pdf

Fondo de las Naciones Unidas para la Infancia. (2005). SEMINARIO INTERNACIONAL: Inclusión Social, Discapacidad y Políticas Públicas. Recuperado de: http://www.unicef.cl/archivos_documento/200/Libro\%20seminario\%20internacional\%20discapacidad.pdf 
Fondo de las Naciones Unidas para la Infancia. (2005). SEMINARIO INTERNACIONAL: Inclusión Social, Discapacidad y Políticas Públicas. Recuperado de: http://www.unicef.cl/archivos_documento/200/Libro\%20seminario\%20internacional\%20discapacidad.pdf

Guirao, I., y Vega, B. (2012). Servicio de Ocio Inclusivo. Cuadernos de buenas prácticas FEAPS. Madrid. FEAPS. Disponible en file:///C:/ Users/Compaq/Downloads/Ocio_Inclusivo.pdf

Madariaga, A., Rubio, I. (s/f). La Inclusión En Ocio De Las Personas Con Discapacidad: Una Línea De Trabajo En El Marco De La Política Social. Deusto, España. Recuperado de: http://www3.uah.es/congresoreps2013/Paneles/panel4/sesion3/aurora.madariaga@deusto. es/TCAurora.pdf

Martínez, M. (2012). Factores contextuales y calidad de vida en las actividades de ocio de los jóvenes y adultos con discapacidades del desarrollo(Tesis doctoral).Universidad de Salamanca, Salamanca, España.

Martínez, M. (2004). Ciencia y Arte de la Metodología Cuialitativa. México: Editorial Trillas. Disponible en: http://doctorado.jairomolina. com.ve/wp-content/uploads/2015/06/Ciencia-y-Arte-en-La-Metodologia-Cualitativa-Martinez-Miguelez-PDF.pdf

Mesa, M. (2014). La influencia del ocio en la calidad de vida de las personas con discapacidad y su relación con el trabajo social(Tesis de pregrado).Universidad de la Laguna, San Cristóbal de La Laguna, España.

Organización Mundial de la Salud. (2001). Clasificación Internacional del Funcionamiento, de la Discapacidad y la Salud. Madrid. Recuperado de: http://ccp.ucr.ac.cr/bvp/pdf/desarrollohumano/omsclasificacion-01.pdf

Organización Mundial de la Salud y Organización Panameicana de la Salud. (2001). Clasificación Internacional del Funcionamiento, de la Discapacidad y la Salud. Madrid. Recuperado de: http://ccp.ucr. ac.cr/bvp/pdf/desarrollohumano/oms-clasificacion-01.pdf

Servicio Nacional de la Discapacidad y Ministerio de Desarrollo Social. (2013). Política Nacional para la Inclusion de las Personas con Discapacidad. Recuperado de http://www.senadis.gob.cl/ descarga/i/1238

Sevilla, C. (2012). "La Animación en el Ocio y Tiempo Libre para Personas con Discapacidad" (Tesis de pregrado). Universidad de Valladolid, España.

Sierra, F. (2011). El Tiempo Libre en las Personas con Discapacidad. Recuperado de https://eala.files.wordpress.com/2011/01/ocio-ydiscapacidad.pdf

Van, A. (2009).Role of Leisure in Stroke Rehabilitation. Oregon: Pacific University CommonKnowledge. Disponible en: http://commons. pacificu.edu/otpf/3 